\title{
In situ optical measurement of liquid drop surface tension in gas metal arc welding
}

\author{
S Subramaniam $\dagger \ddagger$, D R White $\ddagger$, D J Scholl $\S$ and W H Weber $\S$ \\ $\dagger$ Mechanical Engineering and Applied Mechanics, University of Michigan, \\ Ann Arbor, USA \\ $\$$ Manufacturing Systems Department, Ford Research Laboratory, \\ Mail Drop 3135-SRL, PO Box 2053, Dearborn, MI 48121, USA \\ $\S$ Physics Department, Ford Research Laborotary, Mail Drop 3028-SRL, \\ PO Box 2053, Dearborn, MI 48121, USA
}

Received 12 February 1998, in final form 6 May 1998

Abstract. The surface tension of detached liquid drops in pulsed gas metal arc welding was determined in situ from the period of the prolate-oblate oscillations initiated by the detachment event. The oscillating drops were imaged by an optical shadowgraph technique utilizing laser illumination. Images obtained during the welding process were recorded with a high-speed video camera and stored for subsequent analysis. For 4047 aluminium alloy wire, surface tension values in the range $0.54-0.7 \mathrm{~N} \mathrm{~m}^{-1}$ were obtained for various droplet sizes.

\section{Introduction}

The formation and detachment of droplets under the simultaneous influence of plasma, electromagnetic, surface tension and gravitational forces is the fundamental process involved in gas metal arc welding (GMAW). The complex forces that affect the droplets stem primarily from the voltage and current used to maintain the welding plasma and the surface tension of the liquid. However, other factors including viscous drag from the flowing shielding gas, gravity and mechanical vibrations play a role as well. The experimental problems encountered in measuring the properties of droplets during welding are substantial. The bright light and electromagnetic interference radiated from the weld arc plasma, the high temperatures and the rapid formation and detachment of the droplets all contribute to the difficulty of making reliable measurements. There have been numerous attempts to model the welding process, but such models are necessarily highly simplified and approximate and therefore must be validated by careful experiments [1-9].

The present work is directed at one particular aspect of this complex process, the surface tension of the liquid drop. Accurate surface tension values are required in order to estimate the forces on detaching droplets $[2,3,7]$ and model the process of formation and detachment of droplets $[8,9]$. The environmental considerations mentioned above make obtaining these data difficult, particularly since the surface tension is strongly dependent on the temperature, the alloy's composition and the presence of small amounts of surface-active elements such as sulphur and oxygen.
Data on the surface tension of engineering alloys at varying superheats (temperatures above the melting point) are not widely available and typically do not include information on the presence or absence of surface-active species. Even if such data were to hand, they would be difficult to apply, because there is considerable uncertainty regarding the exact temperature of the molten droplets in the welding arc and their local chemical environment. Typically, the surface tension of filler metal droplets has been approximated using estimates of the droplet's temperature and handbook values of surface tension for pure metals [5] or through measurements made by the drop-weight technique under conditions not representative of the actual arc welding environment [10-12]. For these reasons, a technique for measuring the surface tension of droplets in the welding arc for a range of materials under realistic environmental conditions would represent a significant advantage over the methods now in use.

\subsection{Background}

Two different methods have been used to obtain surface tension data in environments representative of arc welding conditions. In the first method, known as the drop-weight technique, a liquid drop is allowed to grow at the end of the wire. As the drop grows, the neck of liquid between the welding wire and the drop continually elongates until it is unable to support the mass of the growing drop [12]. A theoretical relationship known as Tate's law equates the downwards force due to the weight of the drop and the upwards force due to surface tension in the perimeter at 
the supporting tip $[12,13]$. Harkins and Brown [14] have modified Tate's law to account for the shape of the droplet. Ueguri et al [15] and Wen and Lundin [16] employed the drop-weight method to measure the surface tension of welding consumables by using a secondary heat source to produce droplets on arc welding electrodes. Several other researchers $[1,3,9]$ have used the drop-weight method by controlling the welding parameters in a low-current welding arc to produce and detach large droplets (more than twice the diameter of the wire).

Another method for measuring the surface tension of a liquid is by studying waves on its surface. A drop oscillating in its lowest frequency mode of vibration is alternately elongated (prolate) or compressed (oblate) along its axis of symmetry. The liquid drop that detaches from the wire in gas metal arc welding is initially elongated due to electromagnetic forces and after it snaps free from the wire its surface tension causes it to oscillate in exactly this manner. As part of a more general analysis of wave motion [17], Rayleigh derived and verified experimentally the relationship among the surface tension, mass and vibrational period for a free-falling (or levitated) liquid drop. Following Rayleigh, the surface tension $\sigma$ is given by

$$
\sigma=\frac{3 \pi \rho V}{8 \tau^{2}}
$$

where $\rho$ is the density of the liquid, $V$ is the volume of the drop and $\tau$ is the period of oscillation of the lowest frequency (prolate-oblate) mode. In this method of measuring surface tension it is assumed that the equilibrium shape of the droplet is a sphere and the only restoring force is surface tension.

Fraser et al [10] and Keene et al [11] used Rayleigh's equation to determine the surface tension of molten droplets of pure iron, cobalt and nickel. They used an electromagnetic field produced by an induction coil to levitate the droplets in a mixture of argon, helium and hydrogen. However, their work does not consider the effects of typical alloy additions such as $\mathrm{Cu}, \mathrm{Si}, \mathrm{Mn}, \mathrm{Mg}$ and $\mathrm{Zn}$, or tramp elements such as $\mathrm{P}$ and $\mathrm{S}$ present in welding consumables; neither did it reproduce the chemical environment present in gas metal arc welding. In the work presented here, which does not use an induction coil, the only electromagnetic forces present are those produced by the welding voltage and current.

As described below, the liquid drops are imaged during the actual welding process, so the surface tension can be determined in situ from the droplet's volume and period of oscillation. This eliminates the need to characterize and reproduce realistic droplet temperatures and other environmental parameters in a separate apparatus.

\section{Experimental details}

The extreme brightness of the welding-arc plasma discharge makes it impossible to view the metal droplets directly. Instead we used a laser shadowgraph technique, described by Allemand et al [18] and shown schematically in figure 1. The weld region is illuminated with a small $(10 \mathrm{~mW})$ $633 \mathrm{~nm} \mathrm{He}-\mathrm{Ne}$ laser whose output is first expanded to

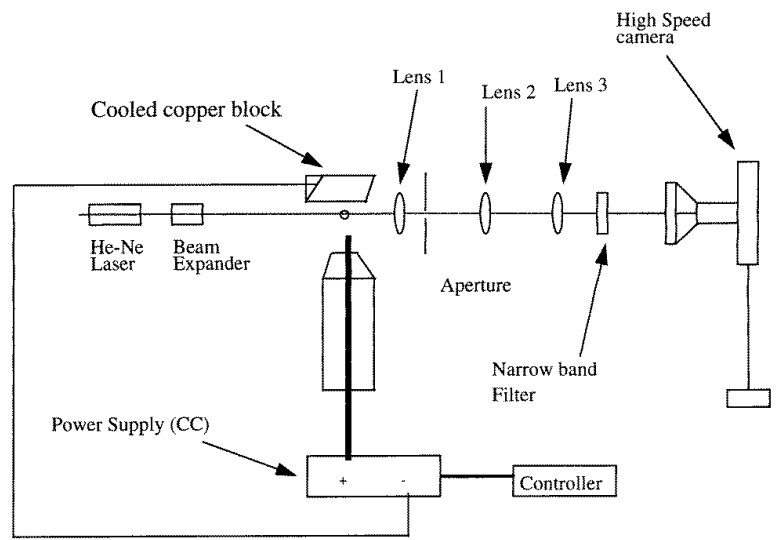

Figure 1. The experimental apparatus for measurement of surface tension.

produce a collimated beam about $2 \mathrm{~cm}$ in diameter. Lens 1 is placed a distance roughly twice its focal length from the weld region and a small aperture is placed precisely at the focal length behind the lens. This aperture serves to pass all the laser light while rejecting most of the arc discharge. Lenses 2 and 3 make the image upright and relay the light efficiently to the camera. A narrowpassband laser-line filter is placed after lens 3 to reject the broadband arc emission too. The welding was videotaped at 4500 frames s $^{-1}$ using a Kodak Ektapro 450 high-speed camera. The images appear as sharp, black shadows of the metal wire and droplets against a red background.

Gas metal arc welding is usually conducted at a constant voltage and current level (CV mode). Under constant conditions, large droplets form if the welding current is low and small droplets are produced if the welding current is high [19]. Small droplets are preferred because their simple vibrational modes can be analysed more accurately than can the complex vibrational behaviour exhibited by the larger droplets. However, since the electromagnetic and plasma forces are much greater at high currents than they are at low currents, the acceleration of the small droplets is also higher, often reaching $400-500 \mathrm{~m} \mathrm{~s}^{-2}$ in welding of aluminium [20]. The field of view from which images can be captured is determined by the distance from the nozzle tip to the workpiece, which is limited to about $5-10 \mathrm{~mm}$ by arc stability. This distance places a constraint on the number of vibrational periods which can be observed by limiting the time interval between the instant of detachment and the instant the drop contacts the weld pool. At higher current levels, which produce higher acceleration (and correspondingly greater velocity and shorter transit time), the constraint is more severe. Since obtaining images of the droplet vibration for the largest possible number of cycles increases the accuracy of measurement, it is desirable to have detachment at low current levels. Unfortunately, these contradictory requirements of a small droplet size (high current) and a low droplet velocity (low current) cannot be simultaneously satisfied with constant voltage and current levels.

In order to obtain small droplets and a low droplet velocity, a variation on the GMAW process known as 
Pulse peak current Pulse transition current

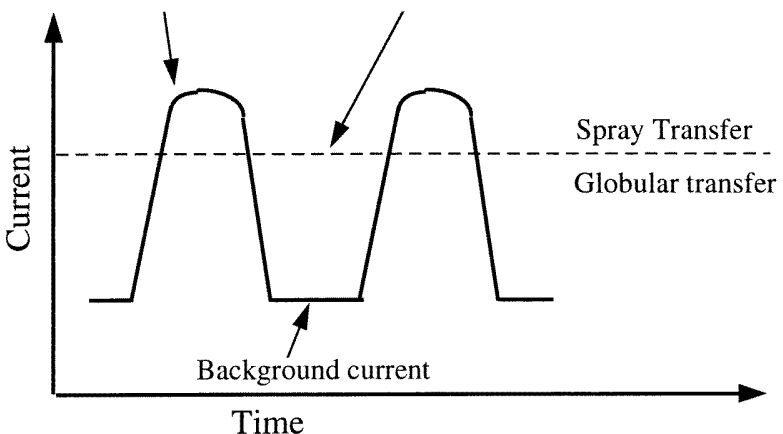

Figure 2. The current waveform in pulsed gas metal arc welding.

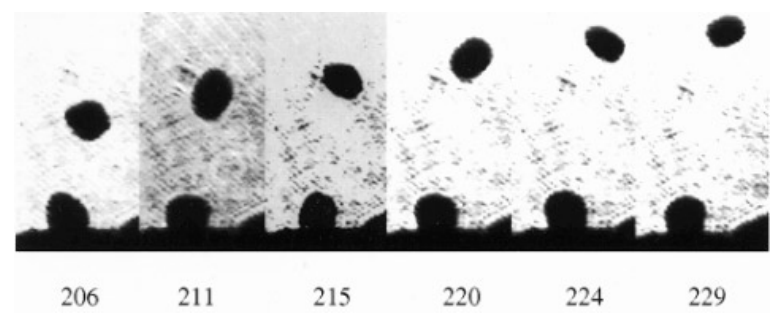

Figure 3. Selected frames showing extremal points of the oscillation of droplet 1.

pulsed gas metal arc welding (PGMAW) was used in these experiments. In PGMAW, as shown in figure 2, the welding power is cycled. By appropriately selecting the peak and background currents, pulse frequency and time at peak current, both the droplet size and the welding current at detachment can be controlled [21]. Small droplets can be detached at low current and velocity. In these experiments, welding was performed in the vertical position, so gravity served to decelerate the detaching droplets further, providing additional time to obtain measurements.

Various combinations of pulsing parameters produce different droplet sizes and velocities. The results presented here were obtained with a peak current of $325 \mathrm{~A}$, a background current of $50 \mathrm{~A}$, a peak current time of $0.9 \mathrm{~ms}$, a background current time of $10 \mathrm{~ms}$ and a wire feeding rate of $5.3 \mathrm{~cm} \mathrm{~s}^{-1}$. These welding parameters are representative of conditions which might be used in an industrial welding application. All welding was carried out using a Miller Maxtron 450 inverter power supply in the constant-current mode. The power supply was controlled by programming a commercially available controller with the desired pulse parameters. A $1.2 \mathrm{~mm}$ diameter 4047 aluminium alloy filler wire was used. Argon shielding gas was supplied at a flow rate of $141 \mathrm{~min}^{-1}$.

\section{Results}

Figures 3-5 show digitized images of three different droplets. The frames are selected to show the extremal points of the droplet oscillations. The period is determined by making a least squares fit to $(x, y)$ pairs, where $x_{i}$ is the

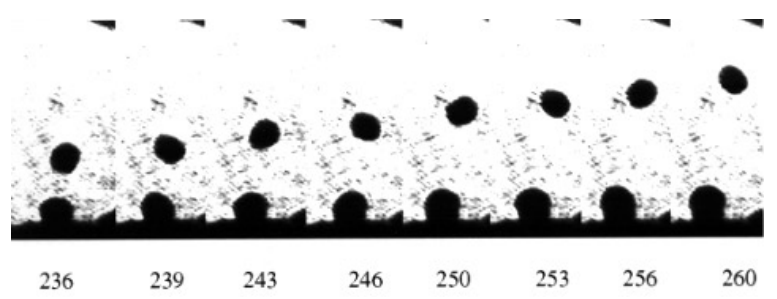

Figure 4. Selected frames showing extremal points of the oscillation of droplet 2.

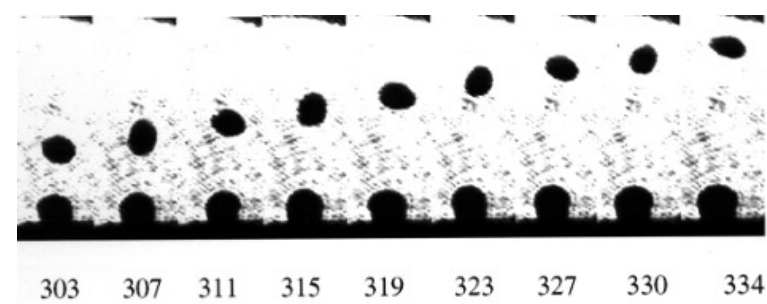

Figure 5. Selected frames showing extremal points of the oscillation of droplet 3.

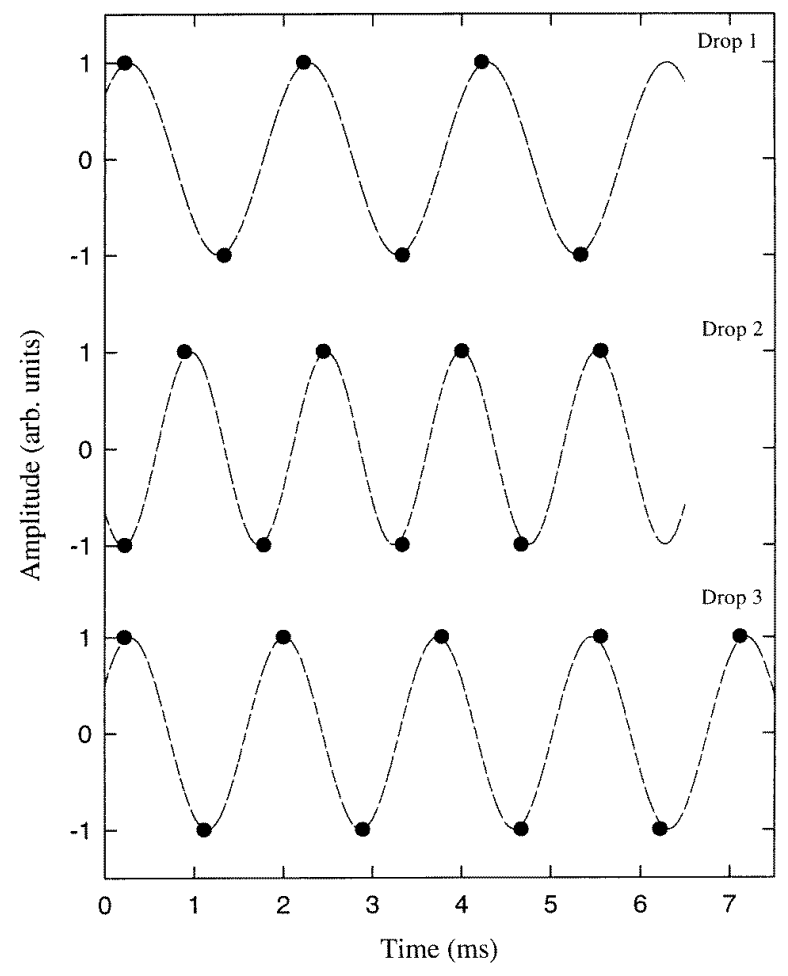

Figure 6. Periodic motion of the three droplets. Points are the extremal experimental values and broken line shows the fitted curve.

time that frame $i$ was recorded and $y_{i}$ is either 1 or -1 for the oblate and prolate extrema, respectively. These points and the fitted sinusoids for the three droplets are shown in figure 6 .

The volume of the droplets was computed by assuming the drop in the frames to be an ellipsoid. The major and minor axis dimensions were measured from the video images using a commercially available image analysis software package. The known wire diameter was used 
Table 1. Computed values of volume, vibrational period and surface tension.

\begin{tabular}{llll}
\hline $\begin{array}{l}\text { Drop } \\
\text { number }\end{array}$ & $\begin{array}{l}\text { Volume } \\
\left(\mathrm{mm}^{3}\right)\end{array}$ & $\begin{array}{l}\text { Time period } \\
(\mathrm{ms})\end{array}$ & $\begin{array}{l}\text { Surface tension } \\
\left(\mathrm{N} \mathrm{m}^{-1}\right)\end{array}$ \\
\hline 1 & 0.92 & 2.0 & $0.54 \pm 0.08$ \\
2 & 0.70 & 1.5 & $0.70 \pm 0.10$ \\
3 & 0.78 & 1.7 & $0.63 \pm 0.09$ \\
\hline
\end{tabular}

to calibrate the length scale. The volume was then computed for several images and the average value was used to calculate the surface tension. The density of the liquid was taken from published values and assumed to be $2000 \mathrm{~kg} \mathrm{~m}^{-3}$ [5]. Although the density of aluminium decreases with increasing temperature in the range 1000$1500 \mathrm{~K}$, the density of aluminium is about one half as sensitive to increasing temperature as is the surface tension.

\section{Discussion}

The calculated surface tension values are quite consistent and are in good agreement with the range of values reported in the literature of $0.6-0.9 \mathrm{~N} \mathrm{~m}^{-1}[5,7,12]$. These results lie at the low end of the published range. This is consistent with the elemental composition of aluminium 4047, which includes 11-13\% silicon and small amounts of iron, copper and magnesium. Low-surface-tension alloys such as aluminium 4047 provide better bead wetting and penetration in welding and better mould filling in casting $[22,23]$.

As noted in table 1 , we estimate the uncertainty in the measurements reported here to be approximately $\pm 15 \%$. This is based on a combination of several factors including the droplet volume, the material's density and the period of oscillation. The uncertainty in volume was estimated from 12 volume measurements (four for each drop). Each measurement was normalized by the mean volume of the corresponding drop, then the standard deviation was calculated for the resulting population of 12 measurements. The estimated uncertainty in density was based on the authors' judgment because it is believed to be primarily due to uncertainty in the droplet temperature, which was not measured. The experimental uncertainty in the period of oscillation is the time resolution (half the time between frames) divided by the total time the droplet was observed. These three sources of error, which were assumed to be uncorrelated, were combined to give the final estimated uncertainty. Some additional assumptions may also affect the results. The Rayleigh formula was derived for oscillations with an amplitude much less than the diameter of the droplet. However, the typical vibrational amplitude is nearly as large as the droplet diameter. Rayleigh also assumed irrotational flow inside the drop, which might not be true in this case. The droplet temperature was assumed to be uniform; because the droplet is small, its thermal conductivity is high and convective flow occurs within it [24], this assumption should be appropriate.

A droplet-size dependence of surface tension has been reported in the literature [25] and in principle could be observed with the technique described here. The reader may notice that the surface tension values shown in table 1 appear to decrease slightly with increasing droplet size. However, this decrease falls within the experimental error and is therefore not significant. The complex, timedependent electromagnetic fields present in the welding plasma will undoubtedly affect the oscillations of droplets to some degree. These effects are assumed to be small and neglected; studies of levitated droplets support this assumption [25-28].

The value of the present experiment is not that this technique is free of approximations and assumptions, but rather that its uncertainties are different from and complementary to those of other methods for measuring surface tension. The ability to account for the effects of alloy composition and chemical environment is a key advantage. A number of surface active elements could be present in the molten metal, and these can dramatically affect surface tension even in small concentrations; sulphur is one example [5]. Sulphur is present as a tramp element in many commercially important materials including welding consumables. Published data, however, typically provide surface tension measurements only for pure metals.

Several gases are used during arc welding, including argon, oxygen, carbon dioxide, helium and their combinations, to which speciality gases such as NO may be added. Some of these are also reported to affect the surface tension of the droplets and the weld pool $[5,9,11,29]$. During the welding process a plasma is formed and spectroscopic measurements on welding arcs have shown that some gaseous species are dissociated or ionized [30]. Furthermore, the plasma contains a significant amount of metal vapour from the welding consumables, with easily vaporized alloying elements such as magnesium particularly prevalent. The level of the welding current also affects the amounts of the various species present in the plasma [5]. These factors and their complex interactions control the composition of the arc atmosphere and their effects on the surface tension of the detaching droplets have not been documented and may be far from negligible.

As a result, in situ measurements provide the most accurate means of accounting for the effects of alloying elements and the plasma's composition on the surface tension of the weld metal. A method used by Chen and Tsamopoulos [31] to determine the surface tension of liquids may also have potential for in situ measurements in a welding environment. They studied the nonlinear dynamics of capillary bridges, obtaining experimental values of the resonance frequency of a standing wave on a capillary bridge. This resonance frequency was then calculated using a finite element analysis solution of the Navier-Stokes equation to determine the surface tension of the liquid. A liquid metal bridge does form between the electrode and the detaching droplet under certain welding conditions, so this approach could conceivably be applied to the welding process. However, the analysis is much more complex than the method considered here and it is not known for certain whether the results would be more accurate.

Improvements to the method presented here could be made and might result in greater accuracy in the resulting 
estimates of the surface tension. The estimates of density values used in these calculations were fairly crude and may be the most significant sources of error. These could be refined through additional experimental studies. Rayleigh considered small-amplitude oscillations in his work. A more accurate model for the droplet shape which is valid for large-amplitude oscillations and includes higher frequency vibrational modes would increase the accuracy of the analysis by addressing inaccuracies in the measurements of the droplet period and volume. A further means for improvement of this method would be to consider the possible effects of irrotational flow in the droplet and effects of electrostatic charge on the droplet. Finally, there are electromagnetic effects mentioned earlier which should be included [24-27]. Despite these recognized areas in which improvements to the model could be made, the results obtained with this simple analysis are clearly consistent with previously reported results and have the advantage of incorporating the effects of composition and environment.

\section{Conclusions}

A simple experimental technique for in situ determination of the surface tension of weld metal droplets in the welding arc has been presented, which yields results consistent with the wide range of published data. The work is based on techniques that have been used widely for determining the surface tension of liquids. Accurate surface tension values are needed for many modelling and analysis applications in welding and this approach handles certain difficulties encountered with other approaches to making accurate assumptions about the effects of alloying elements, the droplet temperature and the plasma composition on the surface tension of weld metal droplets.

\section{References}

[1] Needham J C, Cooksey C J and Milner D R 1960 Metal transfer in inert-gas shielded-arc welding $B r$. Welding $J$. 2 101-14

[2] Amson J C 1962 An analysis of gas-shielded consumable metal arc welding system. Part 1: drop detachment $B r$. Welding J. 9 472-83

[3] Waszink J H and Graat L H J 1983 Experimental investigation of the forces acting on a drop of weld metal Welding J. 62 108-16s

[4] Allum C J 1985 Metal transfer in arc welding as a varicose instability: I. varicose instabilities an a current carrying liquid cylinder with surface charge J. Phys. D: Appl. Phys. 18 1431-46

[5] Lancaster J F 1986 The Physics of Welding (Oxford: Pergamon) pp 32-43

[6] Nemchinsky V A 1994 Size and shape of the liquid droplet at the molten tip of an arc electrode J. Phys. D: Appl. Phys. 27 1433-42

[7] Kim Y S and Eagar T 1993 Analysis of metal transfer in gas metal arc welding Welding J. 72 269-78s

[8] Haidar J and Lowke J J 1996 Predictions of metal droplet formation in arc welding J. Phys. D: Appl. Phys. 29 2951-60
[9] Jones L A 1996 Dynamic electrode forces in gas metal arc welding PhD Dissertation Massachusetts Institute of Technology (Cambridge, MA)

[10] Fraser M E, Lu W K, Hamielec A E and Murarka R 1971 Surface tension measurement on pure liquid iron and nickel by an oscillating drop technique Metall. Trans. 2 817-23

[11] Keene B J, Mills K C and Brooks R F 1985 Surface properties of liquid metals and their effects on weldability Mater. Sci. Technol. 1 568-71

[12] Keene B J 1993 Review of data for the surface tension of pure metals Int. Mater. Rev. 38 157-92

[13] Boucher E A and Evans M J B 1975 Pendent drop profiles and related capillary phenomena Proc. R. Soc. A 346 349-74

[14] Harkins W D and Brown F E 1919 The determination of surface tension (free surface energy), and the weight of falling drops: the surface tension of water and benzene by the capillary height method J. Am. Chem. Soc. 41 499-524

[15] Ueguri S, Hara K and Komura H 1985 Study of metal transfer in pulsed GMAW Welding J. 64 242-50s

[16] Wen J and Lundin C D 1986 Technical note: surface tension of 304 stainless steel under welding conditions Welding J. $65138 \mathrm{~s}$

[17] Rayleigh Lord 1879 On the capillary phenomena of jets Proc. R. Soc. XXIX 71-97

[18] Allemand C D, Schoeder R, Ries D E and Eager T W 1985 A method of filming metal transfer in welding arcs Welding J. 64 45-7

[19] Lesnewich A 1958 Control of melting rate and metal transfer in gas shielded metal arc welding. Part II control of metal transfer Welding J. 37 418-25s

[20] Subramaniam S 1996 Process modelling and analysis for pulsed gas metal arc welding of an aluminum automotive spaceframe $P h D$ Dissertation West Virginia University (Morgantown, WV)

[21] Needham J C and Carter A W 1965 Material transfer characteristics with pulsed current Br. Welding J. 5 $229-41$

[22] Saunders H L 1991 Welding Aluminum: Theory and Practice (Washington, DC: The Aluminum Association) p 3.3

[23] 1990 Metals Handbook. Properties and Selection: Nonferrous Alloys and Special-Purpose Materials vol 2, 10th edn (American Society of Metals) p 145

[24] Nemchinsky V A 1997 Heat transfer in a liquid droplet hanging at the tip of an electrode during arc welding J. Phys. D: Appl. Phys. 30 1120-4

[25] Sauerland S, Eckler K and Egry I 1992 High-precision surface tension measurements on levitated aspherical liquid nickel droplets by digital image processing J. Mater. Sci. Lett. 11 330-3

[26] Cummings D L and Blackburn D A 1991 Oscillations of magnetically levitated aspherical droplets J. Fluid Mech. 224 395-416

[27] Egry I and Sauerland S 1994 Containerless processing of undercooled melts: measurements of surface tension and viscosity Mater. Sci. Eng. A 178 73-6

[28] Sauerland S, Lohofer G and Egry I 1993 Surface tension measurement on levitated liquid metal drops J. Non-crystalline Solids 156-158 833-6

[29] O'Brian 1991 Welding Processes. Welding Handbook vol 2, 8th edn (Miami, FL: American Welding Society) pp 133-6

[30] White D R 1986 Real time control of hydrogen in welding $P h D$ Thesis University of Illinois (Urbana-Champaign)

[31] Chen T Y and Tsamopoulos J 1993 Nonlinear dynamics of capillary bridges: theory J. Fluid Mech. 255 373-409 